\title{
Análisis de Viscocidad en Lubricantes Mediante Diseño Factorial con Tres Factores.
}

\section{Viscosity Analysis in Lubricants through Factorial Design with Three Factors}

\author{
Luis Rojas, Esp ${ }^{1 *}$, Julio Hurtado, $\mathrm{MsC}^{2}$, and Harold A. Rodríguez-Arias, $\mathrm{MsC}^{1}$ \\ ${ }^{1}$ Fundación Tecnológica Antonio de Arévalo, Colombia, luis.rojas@tecnar.edu.co, harold.rodriguez@tecnar.edu.co \\ ${ }^{2}$ Universidad Tecnológica de Bolívar, Colombia,jhurtado@unitecnologica.edu.co \\ *Corresponding autor
}

\begin{abstract}
In the field of statistics, the experimental design becomes an analysis tool to establish the ideal conditions of a very important process and / or product, analysis of variance it is very useful in cases where there are a series of controllable factors and that affect a complex process in order to optimize them, in the specific case, it is sought to give a solution to a lubricants analysis using a factorial design. For the development of the following document, it is proposed to show the analysis performed on lubricant samples by means of a $3 \times 2^{2}$ factorial design with 9 replicas. The analysis was performed on 108 samples, taking into account three different types of lubricants; which were subjected to a temperature of 40 degrees Celsius by kinematic viscocity test. It was observed in the analysis that taking into account the factors that interacted in the design (types of lubricant, parts, brands), only one of them showed significant interaction. The use of the design of factorial experiments is considered pertinent, since this can be useful in making maintenance decisions when buying a specific lubricant brand. The experiment design was carried out with StatGraphics statistical software.
\end{abstract} viscosity.

Keywords-Anova, Factorial design, maintenance, tribology,

Abstract- En el campo de la estadística, el diseño experimental se convierte en una herramienta de análisis para establecer las condiciones ideales de un proceso y/o producto muy importante, el análisis de varianza es muy útil en los casos en que se cuentan con una serie de factores controlables y que afectan un proceso complejo con el objetivo de optimizarlas, en el caso puntual, se busca darle una solución a un análisis de lubricantes utilizando un diseño factorial. Para el desarrollo del siguiente documento se plantea mostrar el análisis realizado a muestras de lubricante mediante diseño factorial $3 \times 2^{2}$ con 9 réplicas. El análisis se realizó a 108 muestras, teniendo en cuenta tres tipos diferentes de lubricantes; los cuales fueron sometidos a una temperatura de 40 grados centígrados mediante prueba de viscosidad cinemática. Se pudo observar en el análisis que teniendo en cuenta los factores que interactuaban en el diseño (tipos de lubricante, partes, marcas), solo uno de ellos mostro interacción significativa. Se plantea pertinente el uso del diseño de experimentos factorial, ya que este puede ser útil en la toma de decisiones de mantenimiento al momento de comprar de una marca de lubricante especifica. El diseño de experimento fue llevado a cabo con el software estadístico StatGraphics.

Keywords- Anova, Diseño factorial, mantenimiento, tribología, viscosidad.

\section{INTRODUCCIÓN}

El diseño estadístico de experimentos según diversos autores tiene como fin último determinar los procedimientos que se deben realizar para obtener conclusiones estadísticamente objetivas sobre un determinado fenómeno, con el fin de concluir sobre los aspectos inciertos del mismo [1].

Un diseño factorial tiene como finalidad estudiar el efecto que un número determinado de factores produce sobre una o varias variables de respuesta [2] [3]. Para este caso de estudio se utilizó un diseño factorial completo, en el cual se toman todas las observaciones aleatoriamente de todas las combinaciones que pueden generarse entre los niveles de los factores de interés, los cuales reciben el nombre de tratamientos y para el presente caso de estudio son de tipo cualitativo; adicionalmente, 9 réplicas fueron tomadas por cada uno de los 12 tratamientos involucrados por el diseño experimental escogido, aun cuando ha sido demostrado que "para los diseño factoriales que involucran menos de cuatro factores por lo regular se corren replicas para tener la potencia necesaria en las pruebas estadísticas sobre los efectos de interés" [1]. Así, el presente experimento tiene 3 efectos principales y 4 efectos de interacción [4].

El trabajo se presentara de la siguiente forma: Se realizara un diseño de experimento, denominado factorial de $3 \times 2^{2}$ con 9 réplicas y 108 corridas bajo una variable respuesta. Para el desarrollo de este experimento se plantearan los niveles de prueba de cada factor en unidades originales, se planteara el modelo estadístico, las hipótesis de interés, la tabla ANOVA del diseño factorial, las interpretaciones de los efectos y las conclusiones del modelo experimental para analizar las interacciones.

Para dar inicio al experimento es importante resaltar que se estarán dando conceptos tales como análisis de lubricante mediante tribología, siendo el análisis de lubricantes una ciencia que se ha desarrollado con los años y que data su importancia en la gerencia de mantenimiento dentro de cualquier industria que trabaje con motores y maquinaria

Digital Object Identifier (DOI): http://dx.doi.org/10.18687/LACCEI2018.1.1.43 ISBN: 978-0-9993443-1-6

ISSN: $2414-6390$

$1^{\text {th }}$ LACCEI International Multi-Conference for Engineering, Education, and Technology: "Innovation in Education and Inclusion”, 19-21 July 2018, Lima, United States. 
industrial de gran tamaño y que necesite lubricar sus partes mecánicas para un óptimo desempeño [5] [6]. Se puede decir que la tribología como ciencia se encarga del estudio de la fricción, lubricación y desgaste de un cuerpo en movimiento contra otro. Los diccionarios definen a la Tribología como la ciencia y tecnología que estudia la interacción de las superficies en movimiento relativo, así como los temas y prácticas relacionadas [7]. La Tribología es el arte de aplicar un análisis operacional a problemas de gran importancia económica, llámese, confiabilidad, mantenimiento, y desgaste del equipo técnico, abarcando desde la tecnología aeroespacial hasta aplicaciones domésticas. El entendimiento de las interacciones superficiales en una interface requiere tener conocimiento de varias disciplinas incluyendo la física, química, matemáticas aplicadas, mecánica de sólidos, mecánica de fluidos, termodinámica, transferencia de calor, ciencia de materiales, lubricación, diseño de máquinas, desempeño y confiabilidad [8].

Cabe resaltar que las muestras tomadas surten de un proceso denominado viscosidad cinemática [9] [10].

\section{METODOLOGIA}

La metodología utilizada para el desarrollo del experimento; fue un diseño experimental de $3 \times 2^{2}$, es decir un diseño con 3 factores, 2 de los cuales tienen dos niveles y uno de tres niveles [2] [3]. En este caso son todos de interés para el analista. Los factores que fueron estudiados; son, el tipo de lubricante, parte de la máquina y marca de la máquina, los cuales son parámetros de un sistema de maquinaria industrial, donde se quiere estudiar si estos tienen efectos significativos sobre la variable de respuesta que en este caso se presenta en términos de la viscosidad que presenta el aceite a una temperatura de $40^{\circ} \mathrm{C}$ para lubricantes industriales luego de 250 horas de uso para el motor y 500 horas de uso para el sistema hidráulico [11] [12] [13].

\section{A. Modelo estadístico del diseño factorial}

El modelo que describe el comportamiento del diseño factorial se puede observar en la ecuación (1).

$\mathrm{yijkl}=\mu+\alpha \mathrm{i}+\beta \mathrm{j}+\gamma \mathrm{k}+(\alpha \beta) \mathrm{ij}+(\alpha \gamma) \mathrm{ik}+(\beta \gamma) \mathrm{jk}+(\alpha \beta \gamma) \mathrm{ijk}+$ Elijk (1)

$\mathrm{i}=1,2, \ldots \mathrm{j}=1,2, \ldots \mathrm{k}=1,2, \ldots \mathrm{l}=1,2, \ldots$

Donde $\mu$ es la media general, $\alpha$ i es el efecto del nivel iesimo del factor A (lubricante), $\beta \mathrm{j}$ es el efecto del nivel $\mathrm{j}$ del factor B (parte de maquinaria: Motor, sistema hidráulico) y $\gamma \mathrm{k}$ es el efecto del nivel $\mathrm{k}$ en el factor $\mathrm{C}$ (marca: SHOVEL, $\mathrm{T}$ Excavador). $(\alpha \beta) \mathrm{ij}+(\alpha \gamma) \mathrm{ik}+(\beta \gamma) \mathrm{jk}$ representan efectos de interacción dobles de dos factores en los niveles ij, ik, jk respectivamente, $\mathrm{y}(\alpha \beta \gamma)$ ijk es el efecto de la interacción triple en la combinación o punto ijk; elijk representa el error aleatorio en la combinación ijkl y 1 son las repeticiones o réplicas del experimento que en total son 9 con 108 corridas experimentales.

\section{B. Hipótesis del diseño factorial}

Teniendo en cuenta el diseño a plantear se anota que las hipótesis, ver ecuación (2) y ecuación (3) en términos de los efectos; muestran que los efectos no son significativos o son iguales a cero como hipótesis nula (H0) y como hipótesis alternativa (HA) diferentes de cero.

$$
\begin{aligned}
& H 0: a 1=a 2=\ldots a i=0 . \\
& H A: a i \neq 0 \text { Para algún } \mathrm{I}
\end{aligned}
$$

\section{Tratamientos del diseño experimental}

Los niveles de prueba para cada factor en unidades originales se muestran en la Tabla I. Los niveles definidos para cada uno de los factores fueron los siguientes: Para el factor tipo de lubricante aceite 15w40 en el nivel alto, 20w50 en el nivel medio y ISO-100 en el nivel bajo, para el factor parte de la maquina se evalúa el motor en nivel alto y el sistema hidráulico en nivel bajo y para marca de la maquina se utiliza SHOVEL y T. ESCAVADOR. Lo anterior se definió luego de llevar a cabo un proceso de prueba con varios niveles en cada factor; adicionalmente analizando los niveles para los cuales se podría tomar muestras, teniendo en cuenta los costos y tiempo de experimento.

El diseño que finalmente se analizó, fue un diseño en el cual se tuvieron en cuenta 9 réplicas por tratamiento. Esto se decidió luego de hacer experimentos con diseños de 2 réplicas y 3 respectivamente y considerar que se requerían más datos para obtener una respuesta más certera y menos variable.

TABLA I. NiVELES De PRUEBA DiseÑo

\begin{tabular}{|l|c|c|c|}
\hline \multirow{2}{*}{ Factor } & \multicolumn{3}{|c|}{ Unidades Originales } \\
\cline { 2 - 4 } & Upper Level & Medium Level & Lowest Level \\
\hline $\begin{array}{l}\text { A: Tipo de } \\
\text { Lubricante }\end{array}$ & $15 \mathrm{~W}-40$ & $20 \mathrm{~W}-50$ & ISO - 100 \\
\hline $\begin{array}{l}\text { B: Parte } \\
\text { maquinaria }\end{array}$ & MOTOR & & HIDRAULICO \\
\hline C: Marca & SHOVEL & & TS. \\
& & & ESAVADOR \\
\hline
\end{tabular}

\section{Réplicas del diseño experimental}

El experimento se realizó con la intención de determinar qué factores influyen sobre la calidad del aceite, para determinar en cuales piezas y para que marcas el lubricante tiene una mayor duración antes de requerir ser cambiado. Es importante aclarar que nos interesa verificar que interacciones entre los factores generan un efecto significativo en nuestras variables de respuesta. Lo anterior puede suministrar

16 $^{\text {th }}$ LACCEI International Multi-Conference for Engineering, Education, and Technology: "Innovation in Education and Inclusion", 19-21 July 2018, Lima, United States. 
información valiosa en decisiones gerenciales para la reducción de costos en mantenimiento de tipo preventivo, correctivo y en la compra de mejores insumos para el proceso de lubricación de las partes de la maquinaria.

Teniendo en cuenta lo anterior; se procede a realizar el análisis del diseño de experimentos. Lo anterior con el fin de determinar el cumplimiento de las hipótesis planteadas en términos de si los factores y sus interacciones tienen efectos significativos sobre la variable de respuesta.

En la Tabla II. Se observan los 12 tratamientos que tiene el diseño experimental con las 9 réplicas por tratamiento, generando un total de 108 corridas experimentales.

TABLA II. TRATAMIENTOS CON REPLICAS

\begin{tabular}{|c|c|c|c|}
\hline $\begin{array}{l}\text { Tipo de } \\
\text { Lubricante }\end{array}$ & Parte Maquina & Marca Pieza & Viscocidad \\
\hline $15 \mathrm{~W} 40$ & MOTOR & SHOVEL & 108,9 \\
\hline $15 \mathrm{~W} 40$ & MOTOR & $\begin{array}{l}\mathrm{T} \\
\text { ESCAVADOR }\end{array}$ & 108,2 \\
\hline $15 \mathrm{~W} 40$ & $\begin{array}{l}\text { S. } \\
\text { HIDRAULICO }\end{array}$ & SHOVEL & 105 \\
\hline $15 \mathrm{~W} 40$ & $\begin{array}{l}\text { S. } \\
\text { HIDRAULICO }\end{array}$ & $\begin{array}{l}\mathrm{T} \\
\text { ESCAVADOR } \\
\end{array}$ & 106,4 \\
\hline 20W50 & MOTOR & SHOVEL & 157,9 \\
\hline 20W50 & MOTOR & $\begin{array}{l}\text { T } \\
\text { ESCAVADOR }\end{array}$ & 149,9 \\
\hline 20W50 & $\begin{array}{l}\text { S. } \\
\text { HIDRAULICO }\end{array}$ & SHOVEL & 157,9 \\
\hline 20W50 & $\begin{array}{l}\text { S. } \\
\text { HIDRAULICO }\end{array}$ & $\begin{array}{l}\mathrm{T} \\
\text { ESCAVADOR } \\
\end{array}$ & 149,9 \\
\hline ISO-100 & MOTOR & SHOVEL & 102,3 \\
\hline ISO-100 & MOTOR & $\begin{array}{l}\mathrm{T} \\
\text { ESCAVADOR }\end{array}$ & 103,4 \\
\hline ISO-100 & $\begin{array}{l}\text { S. } \\
\text { HIDRAULICO }\end{array}$ & SHOVEL & 102,5 \\
\hline ISO-100 & $\begin{array}{l}\text { S. } \\
\text { HIDRAULICO }\end{array}$ & \begin{tabular}{|l} 
T \\
ESCAVADOR \\
\end{tabular} & 107,3 \\
\hline $15 \mathrm{~W} 40$ & MOTOR & SHOVEL & 104,9 \\
\hline $15 \mathrm{~W} 40$ & MOTOR & $\begin{array}{l}\mathrm{T} \\
\text { ESCAVADOR }\end{array}$ & 109,7 \\
\hline $15 \mathrm{~W} 40$ & $\begin{array}{l}\text { S. } \\
\text { HIDRAULICO }\end{array}$ & SHOVEL & 106,9 \\
\hline $15 \mathrm{~W} 40$ & $\begin{array}{l}\text { S. } \\
\text { HIDRAULICO }\end{array}$ & $\begin{array}{l}\mathrm{T} \\
\text { ESCAVADOR } \\
\end{array}$ & 109,9 \\
\hline 20W50 & MOTOR & SHOVEL & 159,5 \\
\hline 20W50 & MOTOR & $\begin{array}{l}\mathrm{T} \\
\text { ESCAVADOR }\end{array}$ & 157 \\
\hline 20W50 & $\begin{array}{l}\text { S. } \\
\text { HIDRAULICO }\end{array}$ & SHOVEL & 159,5 \\
\hline 20W50 & $\begin{array}{l}\text { S. } \\
\text { HIDRAULICO }\end{array}$ & \begin{tabular}{|l}
$\mathrm{T}$ \\
ESCAVADOR \\
\end{tabular} & 157 \\
\hline ISO-100 & MOTOR & SHOVEL & 105,8 \\
\hline ISO-100 & MOTOR & $\begin{array}{l}\mathrm{T} \\
\text { ESCAVADOR } \\
\end{array}$ & 106,9 \\
\hline ISO-100 & $\begin{array}{l}\text { S. } \\
\text { HIDRAULICO }\end{array}$ & SHOVEL & 105,4 \\
\hline
\end{tabular}

\begin{tabular}{|c|c|c|c|}
\hline ISO-100 & $\begin{array}{l}\text { S. } \\
\text { HIDRAULICO }\end{array}$ & \begin{tabular}{|l}
$\mathrm{T}$ \\
ESCAVADOR
\end{tabular} & 102,7 \\
\hline $15 \mathrm{~W} 40$ & MOTOR & SHOVEL & 107,9 \\
\hline $15 \mathrm{~W} 40$ & MOTOR & \begin{tabular}{|l} 
T \\
ESCAVADOR
\end{tabular} & 100,3 \\
\hline $15 \mathrm{~W} 40$ & $\begin{array}{l}\text { S. } \\
\text { HIDRAULICO }\end{array}$ & SHOVEL & 102,2 \\
\hline $15 \mathrm{~W} 40$ & $\begin{array}{l}\text { S. } \\
\text { HIDRAULICO }\end{array}$ & \begin{tabular}{|l|}
$\mathrm{T}$ \\
ESCAVADOR \\
\end{tabular} & 106,3 \\
\hline 20W50 & MOTOR & SHOVEL & 162,2 \\
\hline 20W50 & MOTOR & \begin{tabular}{|l|}
$\mathrm{T}$ \\
ESCAVADOR \\
\end{tabular} & 129,8 \\
\hline 20W50 & $\begin{array}{l}\text { S. } \\
\text { HIDRAULICO }\end{array}$ & SHOVEL & 162,2 \\
\hline 20W50 & $\begin{array}{l}\text { S. } \\
\text { HIDRAULICO }\end{array}$ & \begin{tabular}{|l|}
$\mathrm{T}$ \\
ESCAVADOR
\end{tabular} & 158,8 \\
\hline ISO-100 & MOTOR & SHOVEL & 105,7 \\
\hline ISO-100 & MOTOR & \begin{tabular}{|l|} 
T \\
ESCAVADOR
\end{tabular} & 104,5 \\
\hline ISO-100 & $\begin{array}{l}\text { S. } \\
\text { HIDRAULICO }\end{array}$ & SHOVEL & 103,9 \\
\hline ISO-100 & $\begin{array}{l}\text { S. } \\
\text { HIDRAULICO }\end{array}$ & \begin{tabular}{|l}
$\mathrm{T}$ \\
ESCAVADOR \\
\end{tabular} & 106,6 \\
\hline $15 \mathrm{~W} 40$ & MOTOR & SHOVEL & 96,1 \\
\hline $15 \mathrm{~W} 40$ & MOTOR & \begin{tabular}{|l|}
$\mathrm{T}$ \\
ESCAVADOR
\end{tabular} & 98,7 \\
\hline $15 \mathrm{~W} 40$ & $\begin{array}{l}\text { S. } \\
\text { HIDRAULICO }\end{array}$ & SHOVEL & 106,6 \\
\hline $15 \mathrm{~W} 40$ & $\begin{array}{l}\text { S. } \\
\text { HIDRAULICO }\end{array}$ & \begin{tabular}{|l|}
$\mathrm{T}$ \\
ESCAVADOR \\
\end{tabular} & 105,5 \\
\hline 20W50 & MOTOR & SHOVEL & 159,5 \\
\hline 20W50 & MOTOR & \begin{tabular}{|l|}
$\mathrm{T}$ \\
ESCAVADOR \\
\end{tabular} & 158,8 \\
\hline 20W50 & $\begin{array}{l}\text { S. } \\
\text { HIDRAULICO }\end{array}$ & SHOVEL & 159,5 \\
\hline 20W50 & $\begin{array}{l}\text { S. } \\
\text { HIDRAULICO }\end{array}$ & $\begin{array}{l}\mathrm{T} \\
\text { ESCAVADOR }\end{array}$ & 140,6 \\
\hline ISO-100 & MOTOR & SHOVEL & 104,1 \\
\hline ISO-100 & MOTOR & \begin{tabular}{|l|}
$\mathrm{T}$ \\
ESCAVADOR \\
\end{tabular} & 107 \\
\hline ISO-100 & $\begin{array}{l}\text { S. } \\
\text { HIDRAULICO }\end{array}$ & SHOVEL & 104 \\
\hline ISO-100 & $\begin{array}{l}\text { S. } \\
\text { HIDRAULICO }\end{array}$ & \begin{tabular}{|l|}
$\mathrm{T}$ \\
ESCAVADOR \\
\end{tabular} & 109,7 \\
\hline $15 \mathrm{~W} 40$ & MOTOR & SHOVEL & 98,9 \\
\hline $15 \mathrm{~W} 40$ & MOTOR & \begin{tabular}{|l|}
$\mathrm{T}$ \\
ESCAVADOR
\end{tabular} & 103,2 \\
\hline $15 \mathrm{~W} 40$ & $\begin{array}{l}\text { S. } \\
\text { HIDRAULICO }\end{array}$ & SHOVEL & 99 \\
\hline $15 \mathrm{~W} 40$ & $\begin{array}{l}\text { S. } \\
\text { HIDRAULICO }\end{array}$ & \begin{tabular}{|l|} 
T \\
ESCAVADOR \\
\end{tabular} & 109,8 \\
\hline 20W50 & MOTOR & SHOVEL & 147,9 \\
\hline 20W50 & MOTOR & \begin{tabular}{|l|}
$\mathrm{T}$ \\
ESCAVADOR
\end{tabular} & 140,6 \\
\hline 20W50 & $\begin{array}{l}\text { S. } \\
\text { HIDRAULICO }\end{array}$ & SHOVEL & 147,9 \\
\hline 20W50 & $\begin{array}{l}\text { S. } \\
\text { HIDRAULICO }\end{array}$ & $\begin{array}{l}\mathrm{T} \\
\text { ESCAVADOR }\end{array}$ & 157,6 \\
\hline
\end{tabular}

$1^{\text {th }}$ LACCEI International Multi-Conference for Engineering, Education, and Technology: "Innovation in Education and Inclusion", 19-21 July 2018, Lima, United States. 


\begin{tabular}{|c|c|c|c|}
\hline ISO-100 & MOTOR & SHOVEL & 104,8 \\
\hline ISO-100 & MOTOR & $\begin{array}{l}\mathrm{T} \\
\text { ESCAVADOR }\end{array}$ & 103 \\
\hline ISO-100 & $\begin{array}{l}\text { S. } \\
\text { HIDRAULICO }\end{array}$ & SHOVEL & 106,4 \\
\hline ISO-100 & $\begin{array}{l}\text { S. } \\
\text { HIDRAULICO }\end{array}$ & $\begin{array}{l}\mathrm{T} \\
\text { ESCAVADOR }\end{array}$ & 109,5 \\
\hline $15 \mathrm{~W} 40$ & MOTOR & SHOVEL & 109,6 \\
\hline $15 \mathrm{~W} 40$ & MOTOR & $\begin{array}{l}\mathrm{T} \\
\text { ESCAVADOR }\end{array}$ & 105,4 \\
\hline $15 \mathrm{~W} 40$ & $\begin{array}{l}\text { S. } \\
\text { HIDRAULICO }\end{array}$ & SHOVEL & 109,4 \\
\hline $15 \mathrm{~W} 40$ & $\begin{array}{l}\text { S. } \\
\text { HIDRAULICO }\end{array}$ & $\begin{array}{l}\mathrm{T} \\
\text { ESCAVADOR } \\
\end{array}$ & 109 \\
\hline 20W50 & MOTOR & SHOVEL & 167,9 \\
\hline 20W50 & MOTOR & $\begin{array}{l}\mathrm{T} \\
\text { ESCAVADOR }\end{array}$ & 157,6 \\
\hline 20W50 & $\begin{array}{l}\text { S. } \\
\text { HIDRAULICO }\end{array}$ & SHOVEL & 167,9 \\
\hline 20W50 & $\begin{array}{l}\text { S. } \\
\text { HIDRAULICO }\end{array}$ & $\begin{array}{l}\text { T } \\
\text { ESCAVADOR }\end{array}$ & 126,9 \\
\hline ISO-100 & MOTOR & SHOVEL & 105,1 \\
\hline ISO-100 & MOTOR & $\begin{array}{l}\mathrm{T} \\
\text { ESCAVADOR }\end{array}$ & 105,4 \\
\hline ISO-100 & $\begin{array}{l}\text { S. } \\
\text { HIDRAULICO }\end{array}$ & SHOVEL & 106 \\
\hline ISO-100 & $\begin{array}{l}\text { S. } \\
\text { HIDRAULICO }\end{array}$ & \begin{tabular}{|l} 
T \\
ESCAVADOR \\
\end{tabular} & 106,2 \\
\hline $15 \mathrm{~W} 40$ & MOTOR & SHOVEL & 110,3 \\
\hline $15 \mathrm{~W} 40$ & MOTOR & $\begin{array}{l}\mathrm{T} \\
\text { ESCAVADOR } \\
\end{array}$ & 109,7 \\
\hline $15 \mathrm{~W} 40$ & $\begin{array}{l}\text { S. } \\
\text { HIDRAULICO }\end{array}$ & SHOVEL & 105,1 \\
\hline $15 \mathrm{~W} 40$ & $\begin{array}{l}\text { S. } \\
\text { HIDRAULICO }\end{array}$ & \begin{tabular}{|l} 
T \\
ESCAVADOR \\
\end{tabular} & 105 \\
\hline 20W50 & MOTOR & SHOVEL & 159 \\
\hline 20W50 & MOTOR & $\begin{array}{l}\mathrm{T} \\
\text { ESCAVADOR } \\
\end{array}$ & 126,9 \\
\hline 20W50 & $\begin{array}{l}\text { S. } \\
\text { HIDRAULICO }\end{array}$ & SHOVEL & 159 \\
\hline 20W50 & $\begin{array}{l}\text { S. } \\
\text { HIDRAULICO }\end{array}$ & $\begin{array}{l}\text { T } \\
\text { ESCAVADOR } \\
\end{array}$ & 133,7 \\
\hline ISO-100 & MOTOR & SHOVEL & 104,3 \\
\hline ISO-100 & MOTOR & \begin{tabular}{|l} 
T \\
ESCAVADOR \\
\end{tabular} & 105,4 \\
\hline ISO-100 & $\begin{array}{l}\text { S. } \\
\text { HIDRAULICO }\end{array}$ & SHOVEL & 104,6 \\
\hline ISO-100 & $\begin{array}{l}\text { S. } \\
\text { HIDRAULICO }\end{array}$ & $\begin{array}{l}\mathrm{T} \\
\text { ESCAVADOR } \\
\end{array}$ & 100,9 \\
\hline $15 \mathrm{~W} 40$ & MOTOR & SHOVEL & 102,1 \\
\hline $15 \mathrm{~W} 40$ & MOTOR & $\begin{array}{l}\mathrm{T} \\
\text { ESCAVADOR } \\
\end{array}$ & 98,2 \\
\hline $15 \mathrm{~W} 40$ & $\begin{array}{l}\text { S. } \\
\text { HIDRAULICO }\end{array}$ & SHOVEL & 91,4 \\
\hline $15 \mathrm{~W} 40$ & $\begin{array}{l}\text { S. } \\
\text { HIDRAULICO }\end{array}$ & $\begin{array}{l}\mathrm{T} \\
\text { ESCAVADOR } \\
\end{array}$ & 102,4 \\
\hline 20W50 & MOTOR & SHOVEL & 156,4 \\
\hline
\end{tabular}

\begin{tabular}{|c|c|c|c|}
\hline 20W50 & MOTOR & $\begin{array}{l}\mathrm{T} \\
\text { ESCAVADOR } \\
\end{array}$ & 133,7 \\
\hline 20W50 & $\begin{array}{l}\text { S. } \\
\text { HIDRAULICO }\end{array}$ & SHOVEL & 156,4 \\
\hline 20W50 & $\begin{array}{l}\text { S. } \\
\text { HIDRAULICO }\end{array}$ & \begin{tabular}{|l} 
T \\
ESCAVADOR \\
\end{tabular} & 164,3 \\
\hline ISO-100 & MOTOR & SHOVEL & 107,9 \\
\hline ISO-100 & MOTOR & $\begin{array}{l}\mathrm{T} \\
\text { ESCAVADOR } \\
\end{array}$ & 103,4 \\
\hline ISO-100 & $\begin{array}{l}\text { S. } \\
\text { HIDRAULICO }\end{array}$ & SHOVEL & 106,9 \\
\hline ISO-100 & $\begin{array}{l}\text { S. } \\
\text { HIDRAULICO }\end{array}$ & $\begin{array}{l}\mathrm{T} \\
\text { ESCAVADOR } \\
\end{array}$ & 101 \\
\hline $15 \mathrm{~W} 40$ & MOTOR & SHOVEL & 110,8 \\
\hline $15 \mathrm{~W} 40$ & MOTOR & $\begin{array}{l}\mathrm{T} \\
\text { ESCAVADOR } \\
\end{array}$ & 110,1 \\
\hline $15 \mathrm{~W} 40$ & $\begin{array}{l}\text { S. } \\
\text { HIDRAULICO }\end{array}$ & SHOVEL & 94,9 \\
\hline $15 \mathrm{~W} 40$ & $\begin{array}{l}\text { S. } \\
\text { HIDRAULICO }\end{array}$ & $\begin{array}{l}\mathrm{T} \\
\text { ESCAVADOR } \\
\end{array}$ & 110,3 \\
\hline 20W50 & MOTOR & SHOVEL & 166,9 \\
\hline 20W50 & MOTOR & $\begin{array}{l}\mathrm{T} \\
\text { ESCAVADOR } \\
\end{array}$ & 164,3 \\
\hline 20W50 & $\begin{array}{l}\text { S. } \\
\text { HIDRAULICO }\end{array}$ & SHOVEL & 166,9 \\
\hline 20W50 & $\begin{array}{l}\text { S. } \\
\text { HIDRAULICO }\end{array}$ & $\begin{array}{l}\mathrm{T} \\
\text { ESCAVADOR } \\
\end{array}$ & 148,6 \\
\hline ISO-100 & MOTOR & SHOVEL & 104,3 \\
\hline ISO-100 & MOTOR & $\begin{array}{l}\mathrm{T} \\
\text { ESCAVADOR } \\
\end{array}$ & 103,8 \\
\hline ISO-100 & $\begin{array}{l}\text { S. } \\
\text { HIDRAULICO }\end{array}$ & SHOVEL & 105,7 \\
\hline ISO-100 & $\begin{array}{l}\text { S. } \\
\text { HIDRAULICO }\end{array}$ & $\begin{array}{l}\mathrm{T} \\
\text { ESCAVADOR } \\
\end{array}$ & 101 \\
\hline
\end{tabular}

\section{RESULTADOS}

Se resalta que el análisis de resultados se realizó con el software estadístico Statgraphics. Como primera instancia se realiza el análisis de varianza ANOVA [14] [15] [16] [17].

A. Anova

Luego de cargar los datos de las corridas experimentales se decide realizar un primer análisis de varianza ver Tabla III. De este análisis se puede determinar que el factor parte de la maquina no tiene un efecto significativo sobre la variable respuesta viscosidad. Se puede observar que de las interacciones; la que presenta un efecto sobre la viscosidad es la que existe entre el tipo de lubricante y la marca de la máquina.

Se resalta que el software estadístico STATGRAPHICS hace uso de una herramienta denominada StatAdvisor, aclarando que en el procedimiento para el cálculo del ANOVA se ejecuta un análisis de varianza de varios factores para Viscocidad. Se realizan varias pruebas y gráficas para determinar qué factores tienen un efecto estadísticamente

$16^{\text {th }}$ LACCEI International Multi-Conference for Engineering, Education, and Technology: "Innovation in Education and Inclusion", 19-21 July 2018, Lima, United States. 
significativo sobre Viscocidad. También se evalúa la significancia de las interacciones entre los factores, si es que hay suficientes datos. Adicional se dice que las pruebas-F en la tabla ANOVA le permitirán identificar los factores significativos. Es de anotar que para cada factor significativo, las Pruebas de Rangos Múltiples le dirán cuales medias son significativamente diferentes de otras. La Gráfica de Medias y la Gráfica de Interacciones le ayudarán a interpretar los efectos significativos. Las Gráficas de Residuos le ayudarán a juzgar si los datos han violado los supuestos subyacentes al análisis de varianza.

TABLA III

ANÁLISIS De VARIANZA PARA VisCocidAD - SuMA DE CuAdRADOS TIPO III

\begin{tabular}{|l|l|l|l|l|l|}
\hline Fuente & $\begin{array}{l}\text { Suma de } \\
\text { Cuadrado } \\
\text { s }\end{array}$ & Gl & $\begin{array}{l}\text { Cuadrado } \\
\text { Medio }\end{array}$ & $\begin{array}{l}\text { Razón- } \\
\text { F }\end{array}$ & Valor-P \\
\hline $\begin{array}{l}\text { EFECTOS } \\
\text { PRINCIPALES }\end{array}$ & & & & & \\
\hline A:Tipo Lubricante & 56884,9 & 2 & 28442,4 & 639,86 & 0,0000 \\
\hline B:PARTE MAQUINA & 1,84083 & 1 & 1,84083 & 0,04 & 0,8392 \\
\hline C:MARCA MAQUINA & 307,716 & 1 & 307,716 & 6,92 & 0,0099 \\
\hline INTERACCIONES & & & & & \\
\hline AB & 9,995 & 2 & 4,9975 & 0,11 & 0,8938 \\
\hline AC & 1057,68 & 2 & 528,839 & 11,90 & 0,0000 \\
\hline BC & 45,2408 & 1 & 45,2408 & 1,02 & 0,3155 \\
\hline RESIDUOS & 4356,23 & 98 & 44,4514 & & \\
\hline TOTAL (CORREGIDO) & 62663,6 & 107 & & & \\
\hline
\end{tabular}

Todas las razones -F se basan en el cuadrado medio del error residual.

R-Cuadrada $=93,0482$ porciento

R-Cuadrada (ajustada por g.1.) $=92,4098$ porciento

Los valores del R2 nos indican que se tiene un Anova confiable.

La tabla ANOVA descompone la variabilidad de Viscocidad en contribuciones debidas a varios factores. Puesto que se ha escogido la suma de cuadrados Tipo III (por omisión), la contribución de cada factor se mide eliminando los efectos de los demás factores. Los valores-P prueban la significancia estadística de cada uno de los factores. Puesto que 3 valores-P son menores que 0,05 , estos factores tienen un efecto estadísticamente significativo sobre Viscocidad con un 95,0\% de nivel de confianza.

De lo obtenido en el Anova se observa que es posible eliminar dos de las interacciones, ver Tabla IV; y realizar un mejor análisis de cuales niveles de los factores entre lubricante y marca de maquina son los mejores. Además de lo anterior se observó que el factor parte de maquinaria es irrelevante para la viscosidad, con lo cual para elegir el mejor tratamiento se podría utilizar cualquier nivel teniendo en cuenta el factor económico si se quiere.
ANÁLISIS DE VARIANZA PARA VISCOCIDAD - SUMA De CUADRADOS TIPO III

\begin{tabular}{|c|c|c|c|c|c|}
\hline Fuente & $\begin{array}{c}\text { Suma de } \\
\text { Cuadrados }\end{array}$ & Gl & $\begin{array}{c}\text { Cuadrado } \\
\text { Medio }\end{array}$ & $\begin{array}{c}\text { Razón- } \\
\text { F }\end{array}$ & Valor-P \\
\hline $\begin{array}{c}\text { EFECTOS } \\
\text { PRINCIPALES }\end{array}$ & & & & & \\
\hline A:Tipo Lubricante & 56884,9 & 2 & 28442,4 & 651,19 & 0,0000 \\
\hline $\begin{array}{c}\text { B:B. PARTE } \\
\text { MAQUINA }\end{array}$ & 1,84083 & 1 & 1,84083 & 0,04 & 0,8378 \\
\hline C:MARCA MAQUINA & 307,716 & 1 & 307,716 & 7,05 & 0,0092 \\
\hline INTERACCIONES & & & & & \\
\hline AC & 1057,68 & 2 & 528,839 & 12,11 & 0,0000 \\
\hline RESIDUOS & 4411,47 & 101 & 43,6779 & & \\
\hline TOTAL (CORREGIDO) & 62663,6 & 107 & & & \\
\hline
\end{tabular}

Todas las razones -F se basan en el cuadrado medio del error residual.

\section{R-Cuadrada $=92,9601$ porciento \\ R-Cuadrada (ajustada por g.1.) $=92,5419$ porciento}

Se observa que el valor de R2 aún nos muestra un análisis de varianza confiable y a partir de aquí se realiza un análisis de los gráficos de contrastes, para determinar cuáles son los mejores niveles en cuanto a lubricante y marca de maquinaria se refiere.

\section{B. Análisis de gráficos de contraste}

En el análisis de las gráficas de contraste se tuvieron en cuenta los efectos activos de las interacciones, en este diseño se pudo apreciar que el efecto más importante fue el efecto tipo de lubricante, seguido del efecto marca de la máquina y por último la parte de la maquinaria. Se resalta que la interacción más importante fue la hecha entre el efecto marca de maquinaria y tipo de lubricante y tomando como base la "Fig. 1" se evidencia que el mejor nivel de lubricante es el 20W50, cuando se utiliza en la maquina SHOVEL.

Con base en lo anterior se tendrían los mejores niveles para los factores que generan un efecto en la variable de respuesta (Viscocidad) y como el otro factor parte de la maquina no tiene efecto en la viscosidad del aceite, es indiferente que nivel se utilice.

TABLA IV

Digital Object Identifier: (to be inserted by LACCEI).

ISSN, ISBN: (to be inserted by LACCEI).

16 $^{\text {th }}$ LACCEI International Multi-Conference for Engineering, Education, and Technology: "Innovation in Education and Inclusion", 19-21 July 2018, Lima, United States. 


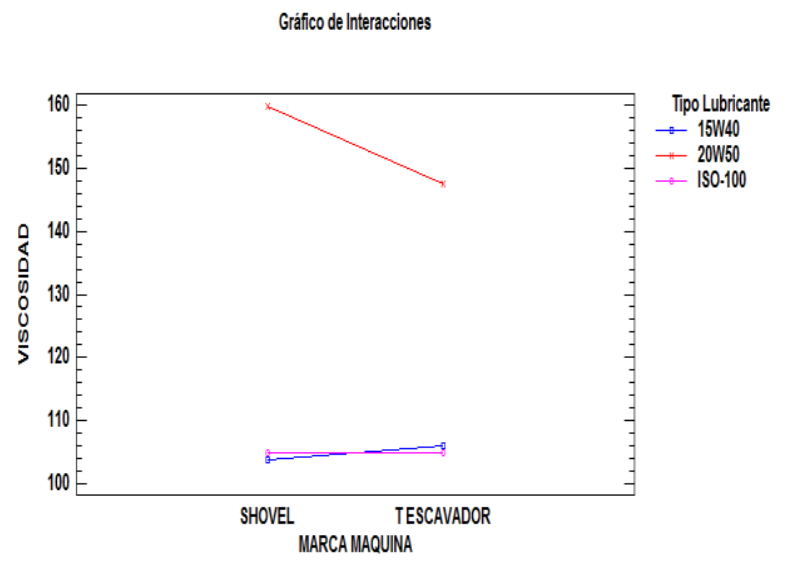

Fig. 1 Grafico de Interacción

\section{Pruebas de múltiples rangos}

Esta tabla aplica un procedimiento de comparación múltiple para determinar cuáles medias son significativamente diferentes de otras. La mitad inferior de la salida muestra las diferencias estimadas entre cada par de medias. El asterisco que se encuentra al lado de los 2 pares indica que estos pares muestran diferencias estadísticamente significativas con un nivel del 95,0\% de confianza. En la parte superior de la página, se han identificado 2 grupos homogéneos según la alineación de las X's en columnas. No existen diferencias estadísticamente significativas entre aquellos niveles que compartan una misma columna de X's. El método empleado actualmente para discriminar entre las medias es el procedimiento de diferencia honestamente significativa (HSD) de Tukey. Con este método hay un riesgo del 5,0\% al decir que uno o más pares son significativamente diferentes, cuando la diferencia real es igual a 0 .

Pruebas de Múltiple Rangos para viscosidad por C Marca Maquina, ver Tabla V.

TABLA V

MÉTOdO 95,0\% PORCENTAJE TUKEY Y HSD

\begin{tabular}{|l|l|l|l|l|}
\hline C:Marca Maquina & Casos & Media LS & Sigma LS & Grupos Homogéneos \\
\hline T ESCAVADOR & 54 & 119,478 & 0,907289 & $\mathrm{X}$ \\
\hline SHOVEL & 54 & 122,854 & 0,907289 & $\mathrm{X}$ \\
\hline
\end{tabular}

\begin{tabular}{|l|l|l|l|}
\hline Contraste & Sig. & Diferencia & +/- Limites \\
\hline SHOVEL - T ESCAVADOR & $*$ & 3,37593 & 2,54628 \\
\hline
\end{tabular}

* indica una diferencia significativa.

Pruebas de Múltiple Rangos para viscosidad por A Lubricante, ver Tabla VI.

TABLA VI
MÉTOdo 95,0\% PORCENTAJE TUKEY Y HSD

\begin{tabular}{|l|l|l|l|l|}
\hline A Lubricante & Casos & Media LS & Sigma LS & $\begin{array}{l}\text { Grupos } \\
\text { Homogéneos }\end{array}$ \\
\hline ISO-100 & 36 & 104,928 & 1,1112 & X \\
\hline 15W40 & 36 & 104,947 & 1,1112 & X \\
\hline 20W50 & 36 & 153,622 & 1,1112 & X \\
\hline
\end{tabular}

\begin{tabular}{|l|l|l|l|}
\hline Contraste & Sig. & Diferencia & $+/$ - Límites \\
\hline 15W40 - 20W50 & $*$ & $-48,675$ & 3,73992 \\
\hline 15W40 - ISO-100 & & 0,0194444 & 3,73992 \\
\hline 20W50 - ISO-100 & $*$ & 48,6944 & 3,73992 \\
\hline
\end{tabular}

* indica una diferencia significativa.

Realizando el analisis de las pruebas de multiples rangos para los dos factores principals relevantes: Tipo lubrcante y marca mquina, se puede anotar que se obtienen los mismos niveles que realizando el analisis en conjutno en el grafico de interaccion. Ver "Fig. 2" y "Fig. 3".

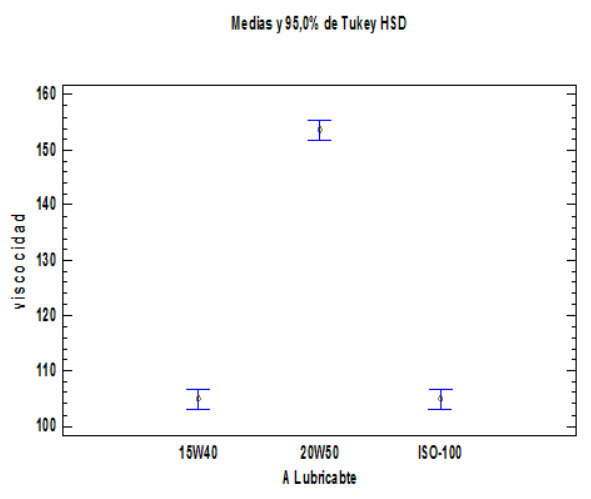

Fig. 2 Grafico de medias Tukey 95\% HSD

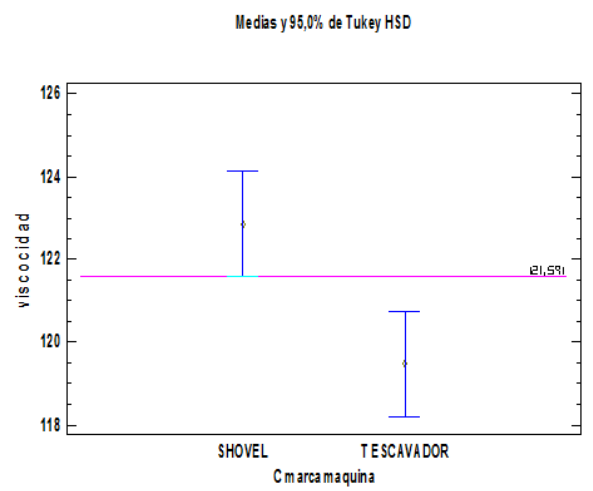

Fig. 3 Grafico de medias Tukey 95\% HSD

Nuevamente se recalca que para el estudio se podria usar cualquier nivel del factor $\mathrm{B}$, teniendo en cuenta el factor

$16^{\text {th }}$ LACCEI International Multi-Conference for Engineering, Education, and Technology: "Innovation in Education and Inclusion", 19-21 July 2018, Lima, United States. 
economico si se quiere, dado que estadisticamente no hay diferencia significativa entre las medias en sus dos diferentes niveles.

\section{CONCLUSIONES}

Se puede apreciar la potencia e importancia de los diseños de experimentos en la toma de decisiones a nivel de procesos industriales. Decisiones relacionadas con la reducción de costos, mediante la realización de compras y escogencia de los productos e insumos para un proceso que minimicen costos y maximicen el tiempo en el cual debe realizarse un mantenimiento como en el caso del experimento presentado.

Puntualmente los resultados obtenidos radica en que los factores principales: Tipo de lubricante y marca de la máquina, generan un efecto sobre la viscosidad del aceite y que la interacción entre estos también. De aquí se logró determinar mediante un análisis de grafico de contrastes cuales son los mejores niveles de dichos factores que maximizan la viscosidad del aceite.

En cuanto al factor parte de la máquina que no fue significativo, este factor era importante de estudiarse, porque se requería saber si la viscosidad el aceite variaba dependiendo de la parte que se estudiara, con el fin de determinar si los mantenimientos y las compras de los lubricantes debían realizarse en tiempos distintos o podían darse en conjunto.

\section{POSIBILIDAD DE MEJORA}

Es considerable la posibilidad de ampliar los niveles del factor parte de la máquina, ya que el resultado obtenido solo se obtuvo involucrando dos de estos. Sin embargo lo anterior fue debido a costos y tiempos en la toma de datos. Si es posible realizar el estudio aumentando estos niveles, se podría constatar con más precisión que la parte de la maquina no genera un efecto sobre la viscosidad del aceite.

\section{REFERENCIAS}

[1] Pulido, H. G., De la Vara Salazar, R., González, P. G., Martínez, C. T., \& Pérez, M. D. C. T. (2004). Análisis y diseño de experimentos. McGraw-Hill

[2] Montgomery, D. C. (1991). Disenoy análisis de experimentos. Grupo Editorial Iberoamericana, Mexico.

[3] Kuehl, R. O. R. O. (2001). Diseño de experimentos: principios estadísticos para el diseño y análisis de investigaciones. Thomson Learning,

[4] Montgomery, D. C. (1991). Disenoy análisis de experimentos. Grupo Editorial Iberoamericana, Mexico.

[5] Martínez, F. (2002). La tribología: ciencia y técnica para el mantenimiento. Editorial Limusa.

[6] Linares, O. O., \& de la Tribología, F. (2005). Tribología y mantenimiento proactivo. Widman International SRL Santa Cruz, Bolivia.

[7] Brostow, W., Deborde, J. L., Jaklewicz, M., \& Olszynski, P. (2003). Tribología con énfasis en polímeros: fricción, resistencia al rayado y al desgaste. Journal of Materials Education, 24(4-6), 119-132.
[8] Flores, M., \& Obed, D. (2017). Análisis de desgastes mecánicos por tribología para reducir costos de mantenimiento del motor de tractor sobre orugas D6T-Caterpillar.

[9] Streeter, V. L., Wylie, E. B., Bedford, K. W., \& Saldarriaga, J. G. (1963). Mecánica de los fluidos (No. QA901. S87 1971.). Ediciones del Castillo.

[10] Martínez, M. M. (2005). Combustión y quemadores (No. 1). Marcombo.

[11] Altmann, C. (2005). El analisis de aceite como herramienta del mantenimiento proactivo en flotas de maquinaria pesada. Consorcio Ambiental de la plata.

[12] Terradillos, J. (2003). Todo sobre la lubricacion de las turbinas y su mantenimiento a través del análisis de aceite. Ingenieria y gestión del mantenimiento: Una nueva visión del mantenimiento. 32, 59-67.

[13] Gutierrez, M., Izquierdo J. F. I., Cadena, X., \& Santiana, G. (2017). Analisis de las vibraciones de un motor ciclo Otto con una mexcla de combustible a base de gasolina y etanol. INNOVA Research Journal , 2(10), 138-146.

[14] Plonsky, L. \& Oswald, F. L. (2017). Multiple regression as a flexible alternative to ANOVA in L2 Research. Studies in second language acquisition, 39(3), 579-592.

[15] Esfe, M. H., Rostamian, H., Shabani-samghabadi, A., \& Arani, A. A. A. (2017). Application of three-level general factorial design approach for thermal conductivity of $\mathrm{MgO}$ /water nanofluids, Applied Thermal Energy, 127, 1194-1199.

[16] Jiménez-Maldonado, A., Ying, Z., Byun, H. R., \& Gomez-Pinilla, F. (2018). Short-term fructose ingestion affects the brain independently from establishment of metabolic syndrome. Biochimica et Biophysica Acta (BBA)-Molecular Basis of Disease, 1864(1), 24-33.

[17] Vengatesan, K., Mahajan, S. B., Sanjeevikumar, P., \& Moin, S. (2018). The Performance Enhancement of Statistically Significant Bicluster Using Analysis of Variance. In Advances in Systems, Control and Automation (pp. 671-678). Springer, Singapore.

16 $^{\text {th }}$ LACCEI International Multi-Conference for Engineering, Education, and Technology: "Innovation in Education and Inclusion", 19-21 July 2018, Lima, United States. 